\title{
NEAT - NMR Spectroscopy for the Estimation of Activity Coefficients of Target Components in Poorly Specified Mixtures
}

\author{
Fabian Jirasek, ${ }^{*, \dagger}$ Jakob Burger, ${ }^{\ddagger}$ and Hans Hasse ${ }^{\dagger}$ \\ $\dagger$ Laboratory of Engineering Thermodynamics (LTD), Technische Universität Kaiserslautern \\ (TUK), Erwin-Schrödinger-Straße 44, 67663 Kaiserslautern, Germany \\ $\ddagger$ Chair of Chemical Process Engineering, Technical University of Munich, Campus \\ Straubing for Biotechnology and Sustainability, Schulgasse 16, 94315 Straubing, Germany \\ E-mail: fabian.jirasek@mv.uni-kl.de
}

\begin{abstract}
Mixtures of which the composition is not fully known are important in many fields of engineering and science, e.g. in biotechnology. Due to the lacking information on the composition, such mixtures cannot be described with common thermodynamic models. In the present work, a method is described with which this obstacle can be overcome for an important class of problems. The method enables the estimation of the activity coefficients of target components in poorly specified mixtures and is based on a combination of NMR spectroscopy with a thermodynamic group contribution method. It is therefore called the NEAT method (NMR spectroscopy for the Estimation of Activity coefficients of Target components in poorly specified mixtures). In NEAT, NMR spectroscopy is used to obtain information on the concentrations of chemical groups in the mixture. The elucidation of the speciation is not required, only the target component has to be known. Modified UNIFAC (Dortmund) is applied in the
\end{abstract}


present work as group contribution method, but NEAT can be extended to any other group contribution method. NEAT has been introduced recently by our group in a short communication, in which, however, only the basic ideas were presented. In the present work, NEAT is described in full detail. Different options of using NEAT are discussed and examples for the application of the method are given. They include a variety of aqueous and non-aqueous mixtures. The results show very good agreement of the activity coefficients that are predicted by NEAT with the corresponding results for the fully specified mixtures.

\section{Introduction}

In many fields of engineering, such as bioprocess engineering, polymer technology, and petrochemical engineering, mixtures of which the composition is only partially known play an important role. Such mixtures are called 'poorly specified mixtures' in the following. Dealing with such mixtures in process design is challenging as classical thermodynamic models of mixtures cannot be applied - they require a full specification of the composition. Approaches to circumvent this problem have been discussed mainly in the field of petrochemical engineering. ${ }^{1-8}$ A general concept for this is introducing pseudo components. However, choosing these pseudo components and assigning thermodynamic properties to them often turns out to be difficult.

A typical task in process engineering is the separation of target components from complex liquid mixtures. Fluid separation processes are generally based on concentration differences between coexisting phases and are often described using the equilibrium stage concept. Therefore, the design of a fluid separation process to capture and purify a target component from a mixture usually requires the knowledge of the thermodynamic activity of the target component in the mixture from which it is to be separated. Rather than referring to the activity, we will refer to the activity coefficient in the following. With thermodynamic models,

such as NRTL, ${ }^{9}$ UNIQUAC, ${ }^{10,11}$ UNIFAC,${ }^{12-15}$ or COSMO-RS, ${ }^{16,17}$ the activity coefficients 
of target components in mixtures of known composition can be calculated, but these methods cannot be applied to mixtures for which the composition is not completely known, i.e. to poorly specified mixtures.

In a previous work of our group, a new method to estimate the activity coefficient of a target component in a poorly specified mixture was presented and its applicability was demonstrated using some examples. ${ }^{18}$ The new method is called NEAT (NMR spectroscopy for the Estimation of Activity coefficients of Target components in poorly specified mixtures). It is based on a simple idea: even for mixtures for which it is hard or impossible to obtain information on the speciation (i.e. the nature and concentration of the components), it is in most cases simple to obtain or at least estimate the corresponding information on the chemical groups in the mixture. A suitable method for obtaining this information on the chemical groups is NMR spectroscopy. It is used in the present study, but also other group-sensitive analytical methods like IR spectroscopy could be applied. The results on the nature and concentration of the chemical groups in the mixture can be used directly in thermodynamic group contribution methods. Several group contribution methods for estimating activity coefficients have been described in the literature, of which the most highly developed and widely used is the UNIFAC method. ${ }^{12}$ UNIFAC is used in the present study in the version modified UNIFAC (Dortmund). ${ }^{14,15}$ Alternatively, other group contribution methods for activity coefficients, such as a group contribution method version of COSMO-RS ${ }^{19}$ could be used. Furthermore, the idea of NEAT is not only applicable to activity coefficients. It could be applied also to any other thermodynamic property for which group contribution methods are available.

Even if only a combination of one-dimensional NMR spectroscopy with UNIFAC is considered, there are many different ways to put the idea of NEAT into practice. But all of them are based on the same fundamental ideas. In the present work, we described these fundamental ideas and put them into a mathematical form. Furthermore, different ways of applying NEAT are described. The examples include aqueous and non-aqueous mixtures. Also the 
application of NEAT to reactive mixtures is discussed. In all cases, the predictions of the activity coefficients of the target components in the poorly specified mixtures obtained from NEAT are compared to the corresponding results for the fully specified mixtures.

\section{Fundamentals of NEAT}

NEAT was developed for tackling poorly specified mixtures, i.e. mixtures of which information on the composition is only partially available. Let us consider a mixture with a known mass $m$ consisting of $i=1 \ldots N$ components. The $N$ components of the mixture are divided into two sets: a first set of $k=1 \ldots K$ known components and a second set of $u=1 \ldots U$ unknown components, hence:

$$
N=K+U
$$

It is assumed that for the known components $k=1 \ldots K$ the following information is available:

$M_{k}$ the molar mass of the component $k$

$m_{k}$ the mass of the component $k$ in the mixture, or alternatively

$n_{k} \quad$ the mole number of the component $k$ in the mixture

No component-specific information is available for the components $u=1 \ldots U$. Even the number $U$ may be unknown.

The total mass of all unknown components in the mixture $m_{\Sigma u}$ can be calculated from:

$$
m_{\Sigma u}=\sum_{u=1}^{U} m_{u}=m-\sum_{k=1}^{K} m_{k}
$$

Let $g=1 \ldots G$ be the chemical groups in the components that make up the mixture such that each component $i$ can be built by combining groups from that set. It is assumed that the molar mass $M_{g}$ of all groups $g$ is known. The number of groups of type $g$ in one molecule of component $i$ is $\nu_{i, g}$. We assume that $\nu_{k, g}$ is known for all known components $k=1 \ldots K$. 
Let $n_{i, g}$ and $m_{i, g}$ be the mole number and mass of group $g$ in component $i$ in the mixture, respectively. For the known components $k, n_{k, g}$ and $m_{k, g}$ are known:

$$
\begin{gathered}
n_{k, g}=\nu_{k, g} n_{k} \\
m_{k, g}=n_{k, g} M_{g}=\nu_{k, g} n_{k} M_{g}
\end{gathered}
$$

In NEAT, spectroscopic analysis is used to obtain information on the chemical groups in the mixture. In the simplest case, each signal in the recorded spectrum of the mixture corresponds to a single group $g$ in the mixture. Other possible, more complex, relations are described in the following chapter. We first assume that at least one signal in the spectrum can be unambiguously and exclusively assigned to one group $g$ occurring in any known component $k$. Provided that the analysis is quantitative, from this signal, a proportionality factor giving the correlation between signal intensity and group mole number can be calculated. It is assumed that this proportionality factor is the same for all groups, which is usually a good assumption. ${ }^{20-22}$ With this proportionality factor, in turn, the mole numbers $n_{g}$ of all groups $g$ in the mixture can be calculated from the spectrum, if an assignment of signals to groups $g$ is available. The task of finding the assignment of signals to groups $g$ is case-specific and therefore discussed in the following chapter.

From $n_{g}$, the group mole fractions $x_{g}^{(n)}$ of all groups $g$ in the mixture can be calculated:

$$
x_{g}^{(n)}=\frac{n_{g}}{\sum_{g=1}^{G} n_{g}}
$$

Let $m_{\Sigma u, g}$ be the contribution of group $g$ to the total mass of all unknown components in the mixture $m_{\Sigma u}$. The number of $m_{\Sigma u, g}$ can be calculated from:

$$
m_{\Sigma u, g}=n_{g} M_{g}-\sum_{k=1}^{K} \nu_{k, g} n_{k} M_{g}
$$


Hence, the previously unknown components can be summarily characterized by group mass fractions $x_{\Sigma u, g}$ regarding only the unknown components $u=1 \ldots U$ in the mixture:

$$
x_{\Sigma u, g}=\frac{m_{\Sigma u, g}}{m_{\Sigma u}}
$$

Furthermore, with the total mole number of all groups in the mixture $n_{\Sigma g}$ :

$$
n_{\Sigma g}=\sum_{g=1}^{G} n_{g}
$$

and the total mole number of all groups of the known components in the mixture $n_{\Sigma k, \Sigma g}$ :

$$
n_{\Sigma k, \Sigma g}=\sum_{k=1}^{K} \sum_{g=1}^{G} \nu_{k, g} n_{k}
$$

the group mole fractions $x_{\Sigma u, g}^{(n)}$ of all groups $g=1 \ldots G$ in the sum of the unknown components in the mixture can be calculated:

$$
x_{\Sigma u, g}^{(n)}=\frac{n_{\Sigma u, g}}{n_{\Sigma u, \Sigma g}}=\frac{n_{g}-n_{\Sigma k, g}}{n_{\Sigma g}-n_{\Sigma k, \Sigma g}}=\frac{n_{g}-\sum_{k=1}^{K} \nu_{k, g} n_{k}}{\sum_{g=1}^{G} n_{g}-\sum_{k=1}^{K} \sum_{g=1}^{G} \nu_{k, g} n_{k}}
$$

The inspection of $x_{\Sigma u, g}^{(n)}$ may give information on the nature of the components $u=1 \ldots U$. Should there only be one such component $U$, it is expected that the ratios $x_{\Sigma u, g_{1}}^{(n)} / x_{\Sigma u, g_{2}}^{(n)}$ of two arbitrary groups $g_{1}$ and $g_{2}$ are integers and the composition of that previously unknown component regarding its groups is obtained. No information is obtained, however, on its molar mass. Should there be more than one unknown component $u(U>1)$, all groups associated to the unknown components are lumped to one mean unknown component $\tilde{u}$ in NEAT. The molar mass $M_{\tilde{u}}$ of this mean unknown component $\tilde{u}$ has to be estimated. Choosing $M_{\tilde{u}}$ can be considered as choosing a 'representative mean molar mass' of the different unknown components. However, it is shown below, that a crude estimate of $M_{\tilde{u}}$ is sufficient in many cases. Using this estimate, the mole number $n_{\tilde{u}}$ of the mean unknown component 
$\tilde{u}$ in the mixture can be calculated:

$$
n_{\tilde{u}}=\frac{m_{\Sigma u}}{M_{\tilde{u}}}
$$

Only then also the mole factions $x_{k}^{(n)}$ of the known components $k=1 \ldots K$ in the mixture can be calculated:

$$
x_{k}^{(n)}=\frac{n_{k}}{\sum_{k=1}^{K} n_{k}+n_{\tilde{u}}}
$$

Also the number of groups $j$ in one molecule of the mean unknown component $\nu_{\tilde{u}, j}$ can then be calculated:

$$
\nu_{\tilde{u}, g}=\frac{n_{g}-\sum_{k=1}^{K} \nu_{k, g} n_{k}}{n_{\tilde{u}}}
$$

NEAT also works, if one of the groups cannot be analyzed with the selected spectroscopic method. Let that group be the group $G$. Hence, NEAT yields only $g=1 \ldots G-1$ group mole numbers $n_{g}$. The mass $m_{G}$ of the group $G$ can be calculated from:

$$
m_{G}=m-\sum_{g=1}^{G-1} n_{g} M_{g}
$$

Assuming that the molar mass $M_{G}$ of the group $G$ is known, the mole number $n_{G}$ is obtained from:

$$
n_{G}=\frac{m_{G}}{M_{G}}
$$

NEAT can also be applied, if not a single group but one of the components as a whole is not accessible by the selected analytical method (e.g. water by ${ }^{13} \mathrm{C}$ NMR spectroscopy). Let that component be the component $N$. The mass $m_{N}$ of the component $N$ can be calculated from:

$$
m_{N}=m-\sum_{g=1}^{G} n_{g} M_{g}
$$

Assuming that the molar mass $M_{N}$ of the component $N$ is known, the mole number $n_{N}$ is obtained from:

$$
n_{N}=\frac{m_{N}}{M_{N}}
$$


All other quantities can be calculated as described above. Eqs (14) - (17) can also be used for testing the internal consistency of NEAT.

\section{Activities and activity coefficients}

There are different possibilities to define activities and activity coefficients. They are based on different ways of normalization of the chemical potential. Four normalizations are considered in the following. As an example, the chemical potential $\mu_{t}$ of the target component $t$ is regarded:

$$
\begin{array}{rlrl}
\mu_{t} & =\mu_{t}^{0}+R T \ln x_{t}^{(n)} \gamma_{t}, & & \text { Raoult } \\
\mu_{t}=\mu_{t}^{0, \mathrm{H}}+R T \ln x_{t}^{(n)} \gamma_{t}^{*}, & \text { Henry } & \\
\mu_{t}=\mu_{t}^{0, c}+R T \ln \frac{c_{t}}{c_{0}} \gamma_{t}^{*, c}, & c_{t}=\frac{n_{t}}{V}, \quad c_{0}=1 \frac{\mathrm{mol}}{\mathrm{l}} \\
\mu_{t}=\mu_{t}^{0, b}+R T \ln \frac{b_{t}}{b_{0}} \gamma_{t}^{*, b}, & b_{t}=\frac{n_{t}}{m_{1}}, \quad b_{0}=1 \frac{\mathrm{mol}}{\mathrm{kg}}
\end{array}
$$

The previously described procedure yields an estimate for the composition of a poorly specified mixture in mole fractions. Hence, Eqs. (18) and (19) can be applied. Also Eq. (20) can be applied in NEAT, if the total volume $V$ of the poorly specified mixture is known. Eq. (21) is applicable, if not only the amount of the target component $t$ but also that of the reference component 1 in the mixture is known or accessible. In the following, results for activity coefficients normalized according to Eq. (18) are shown.

\section{UNIFAC method}

The group contribution method UNIFAC is used in the present work to estimate the activity coefficient of a target component in a poorly specified mixture with NEAT. In UNIFAC, the activity coefficient of the target component $\gamma_{t}$ is computed as the sum of two parts, the 
combinatorial part $\gamma_{t}^{\mathrm{C}}$ and the residual part $\gamma_{t}^{\mathrm{R}}$ :

$$
\ln \gamma_{t}=\ln \gamma_{t}^{\mathrm{C}}+\ln \gamma_{t}^{\mathrm{R}}
$$

The strength of using UNIFAC in NEAT is that the calculation of the residual part $\gamma_{t}^{\mathrm{R}}$ requires only information on groups that is easily available, i.e. no information on the component speciation is required for calculating $\gamma_{t}^{\mathrm{R}}$.

More specifically, for the calculation of $\gamma_{t}^{\mathrm{R}}$, information on the group mole fractions $x_{g}^{(n)}$ of all groups $g$ in the mixture, which are determined or estimated in NEAT from a spectroscopic analysis, and the related relative van der Waals group surfaces $Q_{g}$ and binary group interaction parameters $a_{g g^{\prime}}$ of the groups $g$ in the mixture, which are reported for a large number of groups in the literature, are required. Additionally, the stoichiometry of the target component $t$, i.e. the coefficients $\nu_{t, g}$, and the temperature have to be known.

The calculation of the combinatorial part $\gamma_{t}^{\mathrm{C}}$, on the other hand, requires information on the mole fractions $x_{i}^{(n)}$ of all components $i$ in the mixture. Hence, only to calculate $\gamma_{t}^{\mathrm{C}}$, the groups of the unknown components $u$ in the mixture have to be lumped to a mean unknown component $\tilde{u}$ and the molar mass $M_{\tilde{u}}$ of $\tilde{u}$ has to be estimated. Furthermore, the calculation of $\gamma_{t}^{\mathrm{C}}$ requires information on the relative van der Waals group surfaces $Q_{g}$ and the relative van der Waals group volumes $R_{g}$ of all groups $g$ in the considered mixture, which are reported in the literature. For a detailed description of the UNIFAC equations, the reader is referred to the original papers. ${ }^{12-15}$

\section{Materials and Methods}

\section{Materials}

Ultra pure water was produced with an ultra-purification system of Merck Millipore (Elix Essential 5). Suppliers and purities of the other chemicals used in this work are given in 
Table 1.

Table 1: Suppliers and purities of chemicals used in this work.

\begin{tabular}{|c|c|c|c|}
\hline Chemical & Formula & Supplier & Purity $^{a}$ \\
\hline acetic acid & $\mathrm{C}_{2} \mathrm{H}_{4} \mathrm{O}_{2}$ & Carl Roth & $\geq 99.90 \%$ \\
\hline acetone & $\mathrm{C}_{3} \mathrm{H}_{6} \mathrm{O}$ & Fisher Chemical & $\geq 99.98 \%$ \\
\hline L-ascorbic acid & $\mathrm{C}_{6} \mathrm{H}_{8} \mathrm{O}_{6}$ & Carl Roth & $\geq 99.00 \%$ \\
\hline 1,4-butanediol & $\mathrm{C}_{4} \mathrm{H}_{10} \mathrm{O}_{2}$ & Acros Organics & $\geq 99.90 \%$ \\
\hline tert-butylhydroquinone & $\mathrm{C}_{10} \mathrm{H}_{10} \mathrm{O}_{2}$ & Merck & $\geq 97.00 \%$ \\
\hline citric acid & $\mathrm{C}_{6} \mathrm{H}_{8} \mathrm{O}_{7}$ & Carl Roth & $\geq 99.95 \%$ \\
\hline cyclohexanone & $\mathrm{C}_{6} \mathrm{H}_{10} \mathrm{O}$ & Sigma Aldrich & $\geq 99.80 \%$ \\
\hline dimethoxymethane & $\mathrm{C}_{3} \mathrm{H}_{8} \mathrm{O}_{2}$ & Sigma-Aldrich & $\geq 99.00 \%$ \\
\hline ethanol & $\mathrm{C}_{2} \mathrm{H}_{6} \mathrm{O}$ & Merck & $\geq 99.90 \%$ \\
\hline formaldehyde & $\mathrm{CH}_{2} \mathrm{O}$ & Carl Roth & $\geq 95.00 \%$ \\
\hline methanol & $\mathrm{CH}_{4} \mathrm{O}$ & Carl Roth & $\geq 99.90 \%$ \\
\hline methyl acetate & $\mathrm{C}_{3} \mathrm{H}_{6} \mathrm{O}_{2}$ & Sigma Aldrich & $\geq 99.80 \%$ \\
\hline methyl oleate & $\mathrm{C}_{19} \mathrm{H}_{36} \mathrm{O}_{2}$ & Sigma Aldrich & $\geq 70.00 \%$ \\
\hline methyl palmitate & $\mathrm{C}_{17} \mathrm{H}_{34} \mathrm{O}_{2}$ & Sigma Aldrich & $\geq 97.00 \%$ \\
\hline methyl stearate & $\mathrm{C}_{19} \mathrm{H}_{38} \mathrm{O}_{2}$ & Sigma Aldrich & $\geq 96.00 \%$ \\
\hline oleic acid & $\mathrm{C}_{18} \mathrm{H}_{34} \mathrm{O}_{2}$ & Sigma Aldrich & $\geq 90.00 \%$ \\
\hline 1-propanol & $\mathrm{C}_{3} \mathrm{H}_{8} \mathrm{O}$ & Sigma Aldrich & $\geq 99.50 \%$ \\
\hline pyridine & $\mathrm{C}_{5} \mathrm{H}_{5} \mathrm{~N}$ & Fisher Chemical & $\geq 99.50 \%$ \\
\hline TMS & $\mathrm{C}_{4} \mathrm{H}_{12} \mathrm{Si}$ & Merck & $99.70 \%$ \\
\hline TMSP-d4 & $\mathrm{NaC}_{6} \mathrm{H}_{9} \mathrm{D}_{4} \mathrm{O}_{2} \mathrm{Si}$ & Deutero & $\geq 98.00 \%$ \\
\hline D-xylose & $\mathrm{C}_{5} \mathrm{H}_{10} \mathrm{O}_{5}$ & Alfa Aeser & $\geq 98.00 \%$ \\
\hline
\end{tabular}

\section{Methods}

\section{Sample preparation and NMR analysis}

Liquid sample mixtures containing different components were prepared gravimetrically in $20 \mathrm{ml}$ glass vessels using a balance of Mettler Toledo (AG204). The total mass of all samples was about $10 \mathrm{~g}$. Quantitative ${ }^{1} \mathrm{H}$ and inverse-gated ${ }^{13} \mathrm{C}$ NMR spectra of each sample were recorded. Therefore, about $1 \mathrm{ml}$ of a sample was transferred to an NMR vial. A small amount 
$(<10 \mathrm{mg}$ ) of sodium 3-(trimethylsilyl)tetradeuteriopropionate (TMSP-d4) was added to all aqueous samples as NMR standard. To all non-aqueous samples, a small amount (< $10 \mathrm{mg})$ of tetramethylsilane (TMS) was added. ${ }^{1} \mathrm{H}$ NMR and ${ }^{13} \mathrm{C}$ NMR spectra were taken using a $400 \mathrm{MHz}$ NMR spectrometer from Bruker (Avance) with a cryogenic probe. The acquisition parameters are summarized in Table 2.

The evaluation of the NMR spectra with the software (MestReNova) from Mestrelab Re-

Table 2: NMR acquisition parameters used in the present work.

\begin{tabular}{l|l|l}
\hline \hline Parameter & ${ }^{1} \mathrm{H}$ NMR & ${ }^{13} \mathrm{C} \mathrm{NMR}$ \\
\hline pulse angle / & 10 & 60 \\
acquisition time / s & $2.0-4.0$ & $1.5-2.5$ \\
spectral width / ppm & 15 & 240 \\
number of scans & $8-16$ & $64-128$ \\
relaxation delay / s & 20 & 45 \\
\hline \hline
\end{tabular}

search included appropriate baseline and phase correction. A chemical shift of 0 ppm was assigned to the TMSP-d4 peak.

\section{Estimation of chemical groups and prediction of activity coefficients}

In the following, the procedure for the identification and quantification of the chemical groups in poorly specified mixtures is described. It is assumed that the target component $t$ and its mass fraction $x_{t}$ and molar mass $M_{t}$ are known. For the given total mass $m$ of a mixture, the mole number $n_{t}$ of $t$ in the mixture can be calculated:

$$
n_{t}=\frac{x_{t} m}{M_{t}}
$$

It is assumed that at least one signal $p$ of the target component $t$ in the NMR spectrum of the mixture is separated from all other signals. This signal $p$ is integrated yielding the area $A_{p}$. With the known mole number $n_{t}$ of $t$ and the known number of NMR-active nuclei $z_{p}$ per molecule of $t$ that contribute to the signal $p$, the NMR proportionality factor $P$ is 
calculated:

$$
P=\frac{A_{p}}{n_{t} z_{p}}
$$

If the mass fraction of the target component is not known, an additional component can be added gravimetrically to the mixture prior to the NMR analysis and used as quantitative standard to calculate $P$ analogously. Alternatively, also the technique of the virtual reference could be used. ${ }^{23}$ It is assumed that $P$ is the same for all signals in the spectrum of a mixture. For nuclei in similar chemical groups, this is usually an excellent assumption. ${ }^{20-22}$ But also for nuclei in strongly differing groups, the differences are typically of the order of a few percent and acceptable for NEAT as an estimation method. The validity of the assumption can be checked using experiments with standards. Results from such experiments could also be used for the calibration of the proportionality constants. Since information on the target component $t$ is available, the chemical groups of $t$ are not included in the estimation process in NEAT and all signals of the target component are eliminated from the NMR spectrum yielding a residual spectrum containing only the signals of the unknown components in the mixture. If there are more known components than the target component, they are treated in the same way as the target component. For the elimination, the position of all signals of the considered known components in the spectrum has to be known, which can be determined from spectroscopic experiments or data bases. However, it is generally not necessary to know the exact position of the signals, they only have to be assigned correctly to spectral regions as described below. The signals of the known components then are subtracted from the sum signal of the entire spectral regions. Therefore, no problems with overlapping signals occur. The residual NMR spectrum is divided in regions of chemical shift and a chemical group is assigned to each region. Hence, all signals that appear in a specific chemical shift region are assumed to originate from the same chemical group. In this work, the chemical groups are selected from groups from the modified UNIFAC (Dortmund) parameter table. The assignment of the groups to the spectral regions is not unique and decisions have to be made. The choice depends primarily on the group contribution method that is used and 
on the NMR-spectroscopic method that is applied. Also a priori information on the type of groups or components in the mixture can be taken into account. NEAT is in general not a fixed algorithm but an approach that can be adapted to specific requirements. Furthermore, it is assumed that the assignment of groups to regions does not have to be fully correct to obtain good results. For example, if an NMR signal is assigned to some polar group $\mathrm{P}$, but is caused partly or entirely by some other chemically similar polar group $\mathrm{P}^{*}$, the resulting error in the prediction of the activity coefficient of the target component may not be large. In the present work, different exemplary assignments were used for ${ }^{13} \mathrm{C}$ and ${ }^{1} \mathrm{H}$ NMR spectra that consider groups containing carbon $(\mathrm{C})$, hydrogen $(\mathrm{H})$, and oxygen $(\mathrm{O})$. These assignments are shown in Tables 3 and 4, respectively.

The signals of groups containing exchanging protons, such as hydroxyl, amino, or thiol groups, in ${ }^{1} \mathrm{H}$ NMR spectroscopy are usually broad and their position and shape may strongly depend on the composition of the mixture. However, these signals can be identified easily and an assignment based only on the position is not necessary. Should they overlap with other signals, deconvolution can be used for quantification. For simplicity, the assignment from Table 4 was used for all predictions based on ${ }^{1} \mathrm{H}$ NMR spectroscopy in the present work.

Table 3: Assignment of ${ }^{13} \mathrm{C}$ NMR chemical shift regions to groups from modified UNIFAC (Dortmund) used in this work for the predictions with NEAT. The mole number of water 'H2O' (in aqueous mixtures) or additional primary hydroxyl groups ' $\mathrm{OH}(\mathrm{P})$ ' (in non-aqueous mixtures) was calculated from the mass balance, cf. Eqs. (16) or (14), respectively.

\begin{tabular}{|c|c|c|}
\hline${ }^{13} \mathrm{C}$ NMR chemical shift region & Group assignment & UNIFAC label \\
\hline $0-60$ & alkyl, carbonyl & '래2', '대2CO' \\
\hline $60-90$ & alcohol & ' $\underline{\mathrm{CH}} 2 '+{ }^{\prime} \mathrm{OH}(\mathrm{P})$ ' \\
\hline $90-150$ & alkenyl & ' $\underline{\mathrm{CH}}=\underline{\mathrm{CH}} '$ \\
\hline $150-180$ & carboxyl & '으' \\
\hline$>180$ & carbonyl & 'CH2드' \\
\hline n.a. & water, hydroxyl & ' $\mathrm{H} 2 \mathrm{O}$ ', 'OH(P)' \\
\hline
\end{tabular}

From the integration over each region and the resulting sum signals, the mole numbers 
Table 4: Assignment of ${ }^{1} \mathrm{H}$ NMR chemical shift regions to groups from modified UNIFAC (Dortmund) used in this work for the predictions with NEAT. The mole number of additional quaternary carbon groups was calculated from the mass balance, cf. Eq. (14).

\begin{tabular}{l|l|l}
\hline \hline${ }^{1} \mathrm{H}$ NMR chemical shift region & Group assignment & UNIFAC label \\
\hline $0-3$ & alkyl & ' $\mathrm{C} \underline{\mathrm{H}}$ ' \\
$3-4.5$ & carboxylate & ' $\mathrm{C} \underline{\mathrm{H}} 2 \mathrm{COO}$ \\
$4.5-6$ & alkenyl & ' $\mathrm{C} \underline{\mathrm{H}}=\mathrm{C} \underline{\mathrm{H}}$ ' \\
$6-7$ & aryl & ' $\mathrm{AC} \underline{\mathrm{H}}$ ' \\
$>7$ & hydroxyl & ' $\mathrm{H}(\mathrm{P})$ ' \\
n.a. & quaternary carbon & 'C' \\
\hline \hline
\end{tabular}

of the chemical groups in the mixture are determined using the proportionality factor $P$ and the number of NMR-active nuclei in each assigned chemical group. It should be noted that the obtained mole numbers of chemical groups depend on the group assignment, in particular for groups with different numbers of NMR-active nuclei. For instance, the signals of 'CH', 'CH2', and 'CH3' groups that are not bonded to different substituents are difficult to distinguish from their position in a ${ }^{1} \mathrm{H}$ NMR spectrum alone. The assignment of ' $\mathrm{CH}$ ' groups to all signals in a specific spectral region leads to a three times larger group mole number than the assignment of 'CH3' groups to the same spectral region. This can lead to errors. However, preexisting knowledge can be used to reduce these errors.

Generally, four fundamental cases for the mapping of UNIFAC groups to spectral regions can be distinguished. For each case, an example for the mapping of groups from the UNIFAC table to ${ }^{13} \mathrm{C}$ NMR chemical shift regions as it was used in this work, cf. Table 3, is described in the following.

In the first case, the UNIFAC group contains NMR-active nuclei and all nuclei show signals in the same spectral region. The selected group is then assigned to all signals in this specific region. An example is the assignment of ' $\mathrm{CH}=\mathrm{CH}$ ' groups to all signals in the chemical shift region ranging from $90-150 \mathrm{ppm}$. In the second case, the UNIFAC group contains active nuclei, but they show signals in different spectral regions. An example for this case is the assignment of a ' $\mathrm{CH} 2 \mathrm{CO}$ ' group. This group leads to signals in two spectral regions: 
in the region $>180 \mathrm{ppm}$, the signals of the ' $\mathrm{CO}$ ' part are expected, and in the region 0 $60 \mathrm{ppm}$, the signals of the 'CH2' part are expected. Consequently, 'CH2CO' group are only assigned, if in both regions, at $>180 \mathrm{ppm}$ and at 0 - $60 \mathrm{ppm}$, signals are present and ' $\mathrm{CH} 2$ ' groups are only assigned to the signals in the region 0 - $60 \mathrm{ppm}$ that exceed the signals in the region $>180 \mathrm{ppm}$. The third case considers UNIFAC groups that do not contain NMRactive nuclei but have an evaluable influence on adjacent groups that contain NMR-active nuclei. An example for this case is the assignment of ' $\mathrm{CH} 2$ ' + 'OH(P)' groups, which build together a primary alcohol group, to the signals in the region 60 - 90 ppm. Hence, although 'OH(P)' groups do not show a signal in ${ }^{13} \mathrm{C}$ NMR spectroscopy, information on the number of ' $\mathrm{OH}(\mathrm{P})$ ' groups can thereby be obtained. Finally, in the fourth case, the UNIFAC groups do not contain NMR-active nuclei and there is no evaluable influence on adjacent groups. Information on these groups can only be obtained from the mass balance as described below. An example for this case is the consideration of additional ' $\mathrm{OH}(\mathrm{P})$ ' or ' $\mathrm{H} 2 \mathrm{O}$ ' groups, if ${ }^{13} \mathrm{C}$ NMR spectroscopy is applied.

With the known molar masses of the assigned chemical groups, the total mass of all groups of the unknown components and, hence, the total mass of the unknown components $m_{\Sigma u}$ can be calculated, which is, in turn, used to calculate the difference $\Delta m$ :

$$
\Delta m=m-m_{t}-m_{\Sigma u}
$$

$\Delta m$ is expected to be positive in those cases in which not all groups in the mixture yield a signal in the NMR spectrum. In the other cases, the absolute numbers for $\Delta m$ are expected to be small compared to the mass $m$. In such cases, the relative deviations $|\Delta m| / m$ were found to be of the order of a few percent in the experiments of the present work. Information on how $\Delta m$ was used in the present work are given in the following: for all aqueous mixtures, which were only studied with ${ }^{13} \mathrm{C}$ NMR spectroscopy in the present work, $\Delta m$ was used to determine the mass of water $m_{\mathrm{w}}$ in the mixture, cf. Table 3 . If non-aqueous mixtures were 
studied with ${ }^{13} \mathrm{C}$ NMR spectroscopy, $\Delta m$ was used to determine the number of additional ' $\mathrm{OH}(\mathrm{P})$ ' groups in the mixture, cf. Table 3. If non-aqueous mixtures were studied with ${ }^{1} \mathrm{H}$ NMR spectroscopy, $\Delta m$ was used to determine the number of additional 'C' groups in the mixture, cf. Table 4. If $\Delta m$ was negative, the mole numbers of the determined groups of the unknown components were reduced to comply with the mass balance in all cases. The ratios of the mole numbers of the groups among each other were thereby kept constant. All determined groups of the unknown components in a poorly specified mixture were lumped to one mean unknown component $\tilde{u}$ in all cases. If not stated otherwise, a molar mass of $M_{\tilde{u}}=150 \mathrm{~g} / \mathrm{mol}$ was thereby assigned to $\tilde{u}$.

For the prediction of the activity coefficients of the target component $t$ with NEAT in the present work, each studied aqueous poorly specified mixture was regarded as ternary mixture containing the target component $t$, one mean unknown component $\tilde{u}$, and water $\mathrm{w}$. A schematic representation of the procedure of NEAT for the application to aqueous poorly specified mixtures as used in this work is given in Figure S1 in the Supporting Information.

Each studied non-aqueous poorly specified mixture was either regarded as binary mixture containing the target component $t$ and one mean unknown component $\tilde{u}$ or as ternary mixture containing, besides $t$ and $\tilde{u}$, a solvent $s$. If a solvent $s$ was considered, its nature was assumed to be known and its mole number in the mixture was calculated from the NMR spectrum using the proportionality factor $P$ and a signal that could unambiguously be assigned to $s$. Hence, the chemical groups of $s$ in the mixture were, in analogy to the groups of $t$, not determined by NMR spectroscopy in NEAT.

\section{Results and Discussion}

In the following, the predictions with NEAT for the poorly specified mixtures are always compared to results that were calculated using information on the full speciation of the mixtures, 
which was always known from the gravimetric sample preparation but not used for the predictions. In the Supporting Information, the results for the composition of all studied poorly specified mixtures and the stoichiometry of the mean unknown components as determined from NMR spectra of the mixtures are summarized. These data were used for obtaining the predictions with NEAT. If not stated otherwise, the results for the fully specified mixtures were calculated with the same version of UNIFAC that was used for the predictions. It should be noted, that no statements on the applicability of UNIFAC for the studied system are made here. Obviously, the quality of the predictions with NEAT cannot exceed the quality of the results of the underlying thermodynamic model for the fully specified mixtures.

\section{Aqueous mixtures}

For applying NEAT to aqueous organic mixtures, ${ }^{13} \mathrm{C}$ NMR spectroscopy is a good choice as water has a very broad and shifting signal in the ${ }^{1} \mathrm{H}$ NMR spectrum. In the following, results for the prediction of the activity coefficient of the target component in aqueous poorly specified mixtures with NEAT based on a single ${ }^{13} \mathrm{C}$ NMR spectrum of each mixture and the group assignment from Table 3 are shown. For the predictions, only information on the target component $t$ was used.

As first example, ternary mixtures containing one known target component $t$, water $\mathrm{w}$, and one unknown component $u$ are considered. In Figure 1, results for the activity coefficient of the target component $(t=$ ascorbic acid) in ternary mixtures containing water $(\mathrm{w})$ and the unknown component ( $u=$ cyclohexanone) as a function of the mass fraction of cyclohexanone are shown. The predictions with NEAT, denoted as black squares, show a very good agreement with the results obtained using information on the full speciation of the mixture calculated with UNIFAC, denoted as black line. The predictions of UNIFAC for the activity coefficients of ascorbic acid are very high. As explained above, in the present context, it does not matter whether this is realistic or not. 
Figure 1 also shows results for the activity coefficient of the target component $(t=1,4$ -

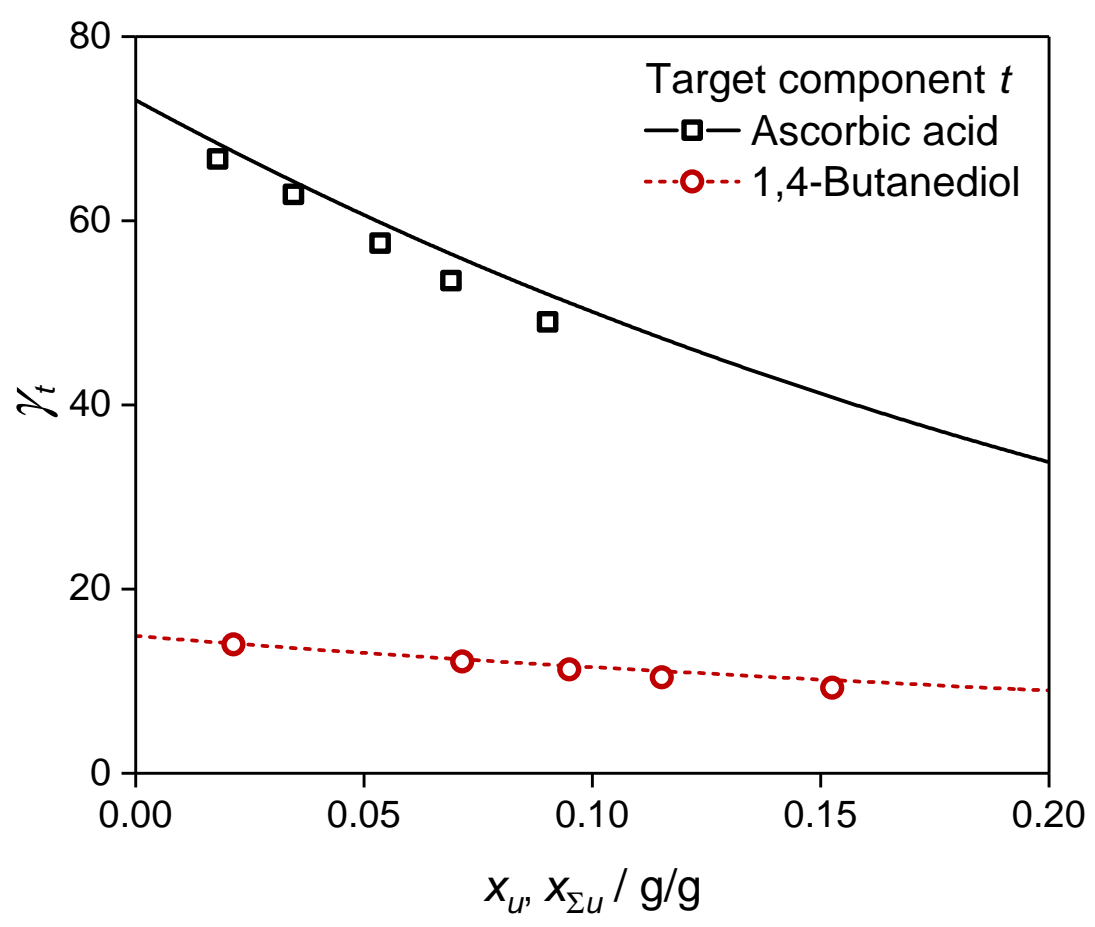

Figure 1: Activity coefficients $\gamma_{t}$ of target component $(t=$ ascorbic acid $)$ in ternary mixtures of $t$ with water $(\mathrm{w})$ and one unknown component ( $u=$ cyclohexanone) and of target component $(t=1,4$-butanediol) in five-component mixtures with water $(\mathrm{w})$ and three unknown components $(u=$ cyclohexanone, pyridine, citric acid; with the same mass ratio in all mixtures) at $298 \mathrm{~K}$ and 1 bar. The mass ratio of $(t: \mathrm{w})$ is $(0.1: 0.9)$ in all mixtures. Lines: results from modified UNIFAC (Dortmund) for the fully specified mixtures. Symbols: predictions with NEAT based on ${ }^{13} \mathrm{C}$ NMR and only information on $t$.

butanediol) in five-component mixtures containing water (w) and three unknown components ( $u=$ cyclohexanone, pyridine, citric acid) as a function of the total mass fraction $x_{\Sigma u}$ of the unknown components. Again, the predictions with NEAT are compared to the results calculated with UNIFAC using information on the full speciation of the mixtures showing an excellent agreement.

As described above, for the predictions with NEAT, the chemical groups of the unknown components $u$ in a poorly specified mixture were lumped to a mean unknown component $\tilde{u}$, to which a molar mass $M_{\tilde{u}}=150 \mathrm{~g} / \mathrm{mol}$ was assigned in the present work. In Figure 2, results for the influence of the value of $M_{\tilde{u}}$ on the predictions with NEAT are shown. As an example, one mixture with the target component $(t=1,4$-butanediol) from Figure 1 was 
selected for this purpose. The results show a very small influence of $M_{\tilde{u}}$ on the predictions

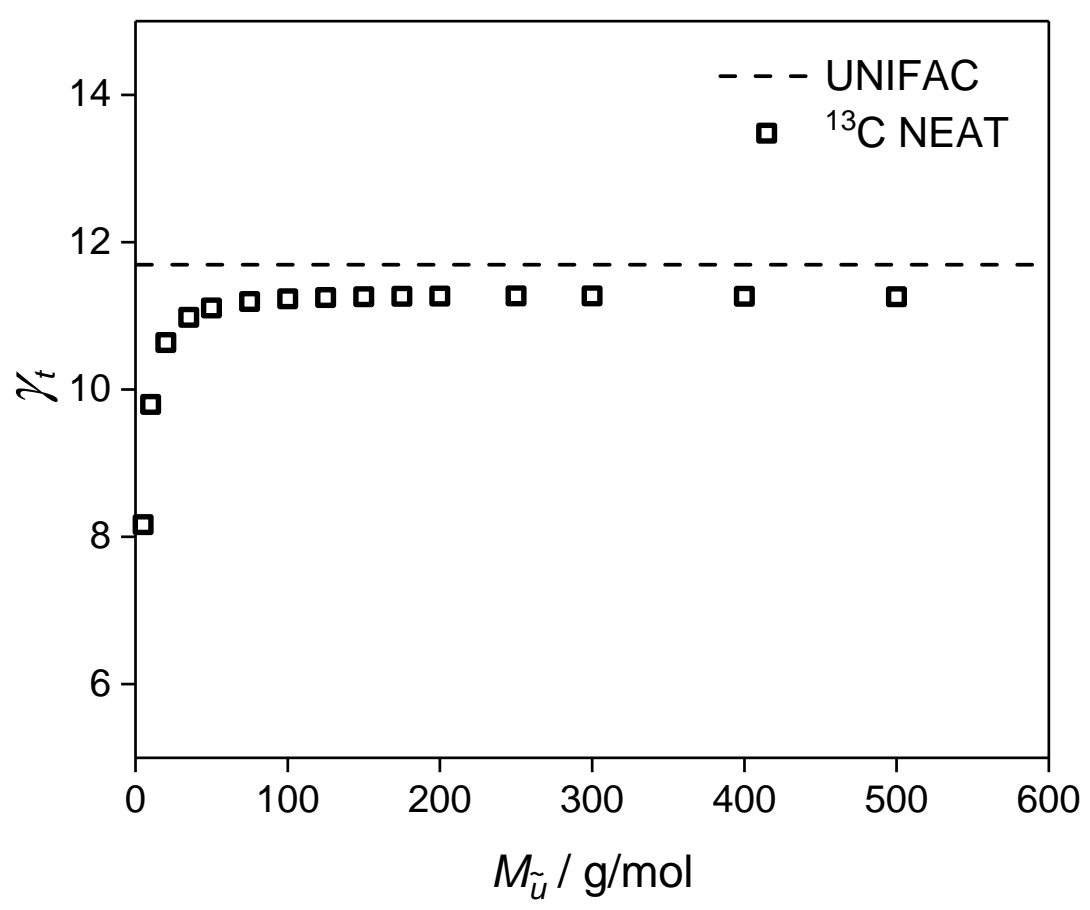

Figure 2: Activity coefficient $\gamma_{t}$ of target component $(t=1,4$-butanediol) in a five-component mixture with water $(\mathrm{w})$ and three unknown components $(u=$ cyclohexanone, pyridine, citric acid; with the same mass ratio) at $298 \mathrm{~K}$ and 1 bar. The composition of the mixture is $x_{t}=0.091 \mathrm{~g} / \mathrm{g}, x_{\Sigma u}=0.095 \mathrm{~g} / \mathrm{g}$. Dashed line: result from modified UNIFAC (Dortmund) for the fully specified mixture. Symbols: predictions with NEAT based on ${ }^{13} \mathrm{C}$ NMR and only information on $t$ for different values of the molar mass $M_{\tilde{u}}$ of the mean unknown component.

with NEAT if no unreasonably small values $\left(M_{\tilde{u}}<50 \mathrm{~g} / \mathrm{mol}\right)$ are chosen. This was found for all studied aqueous mixtures and can be attributed to two points: first, as described above, to the fact that $M_{\tilde{u}}$ does not have an influence on the predominant residual part of the UNIFAC activity coefficient of $t$, but only on the combinatorial part. Second, the concentration of water in the studied mixtures is high. Due to the low molar mass and the resulting high mass fraction of water, the influence of $M_{\tilde{u}}$ on the mole fraction of $t$, which contributes to the combinatorial part of the UNIFAC activity coefficient of $t$, is rather small.

In Figure 3, results for the activity coefficient of the target component $(t=$ acetic acid $)$ in ternary mixtures containing water $(\mathrm{w})$ and one unknown component $(u=x y l o s e)$ are given. In contrast to the previous examples, the predictions with NEAT are compared to results 
calculated with an NRTL model, which was fitted to experimental data on the vapor-liquid equilibrium of the regarded system. ${ }^{24}$ The temperature is therefore in this case not constant to represent the experimental data from Galeotti et al. ${ }^{24}$ For the prediction of the activity coefficients with NEAT, again ${ }^{13} \mathrm{C}$ NMR spectroscopy in combination with modified UNIFAC (Dortmund) was used. The predictions show an excellent agreement with the results from the NRTL model.

NEAT can also be applied to reactive mixtures. Figure 4 shows results for the activity co-

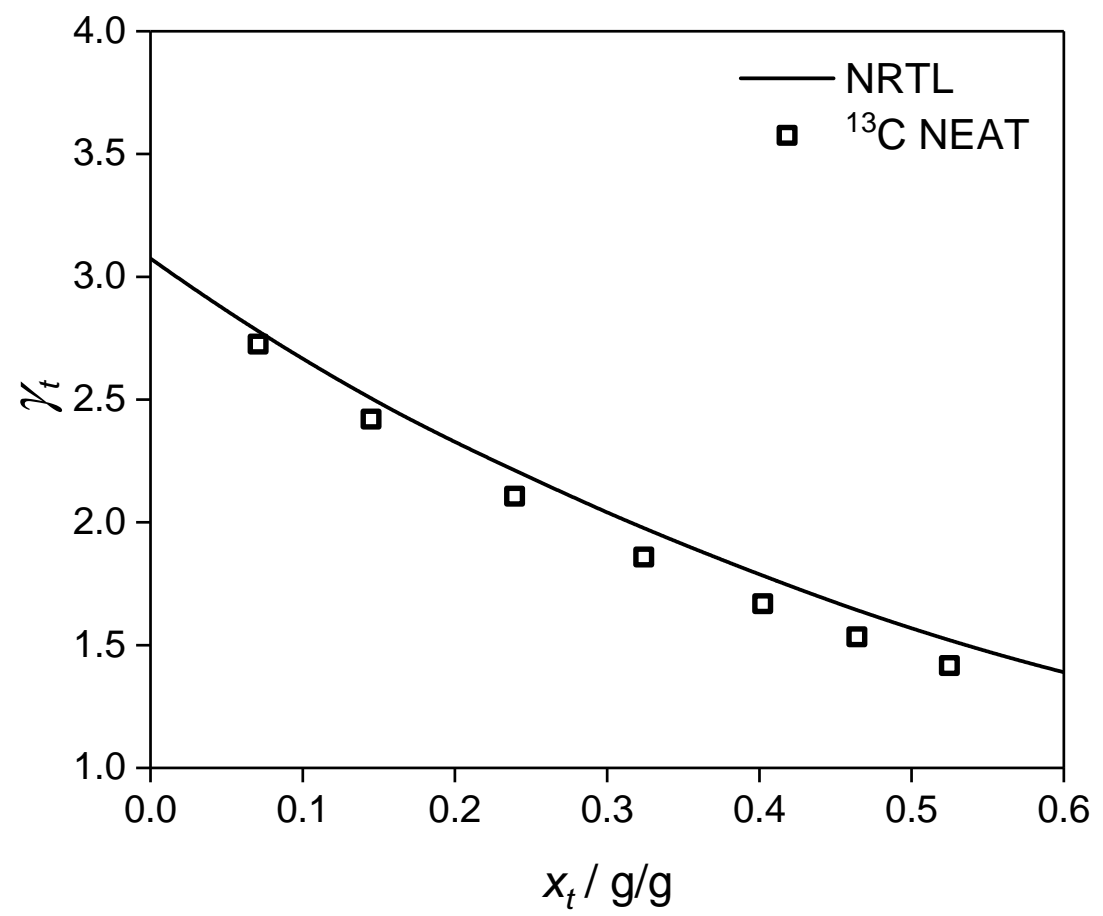

Figure 3: Activity coefficient $\gamma_{t}$ of target component $(t=$ acetic acid) in ternary mixtures with water $(\mathrm{w})$ and one unknown component $(u=$ xylose $)$ at $333.7-335.7 \mathrm{~K}$ and 1 bar. $x_{u}=0.099 \mathrm{~g} / \mathrm{g}$ in all mixtures. Line: results from an NRTL model fitted to experimental data $^{24}$ for the fully specified mixtures. Symbols: predictions with NEAT based on ${ }^{13} \mathrm{C}$ NMR and only information on $t$.

efficient of the target component $(t=$ dimethoxymethane) in mixtures with different overall concentrations of water $(\mathrm{w})$ and the unknown component ( $u=$ formaldehyde). Formaldehyde is reactive in aqueous mixtures and forms polyoxymethylene glycols of different chain lengths with water. ${ }^{25-29}$ Kuhnert et al. ${ }^{25}$ reported an activity-based chemical equilibrium model to describe the formation of polyoxymethylene glycols in aqueous formaldehyde-containing 


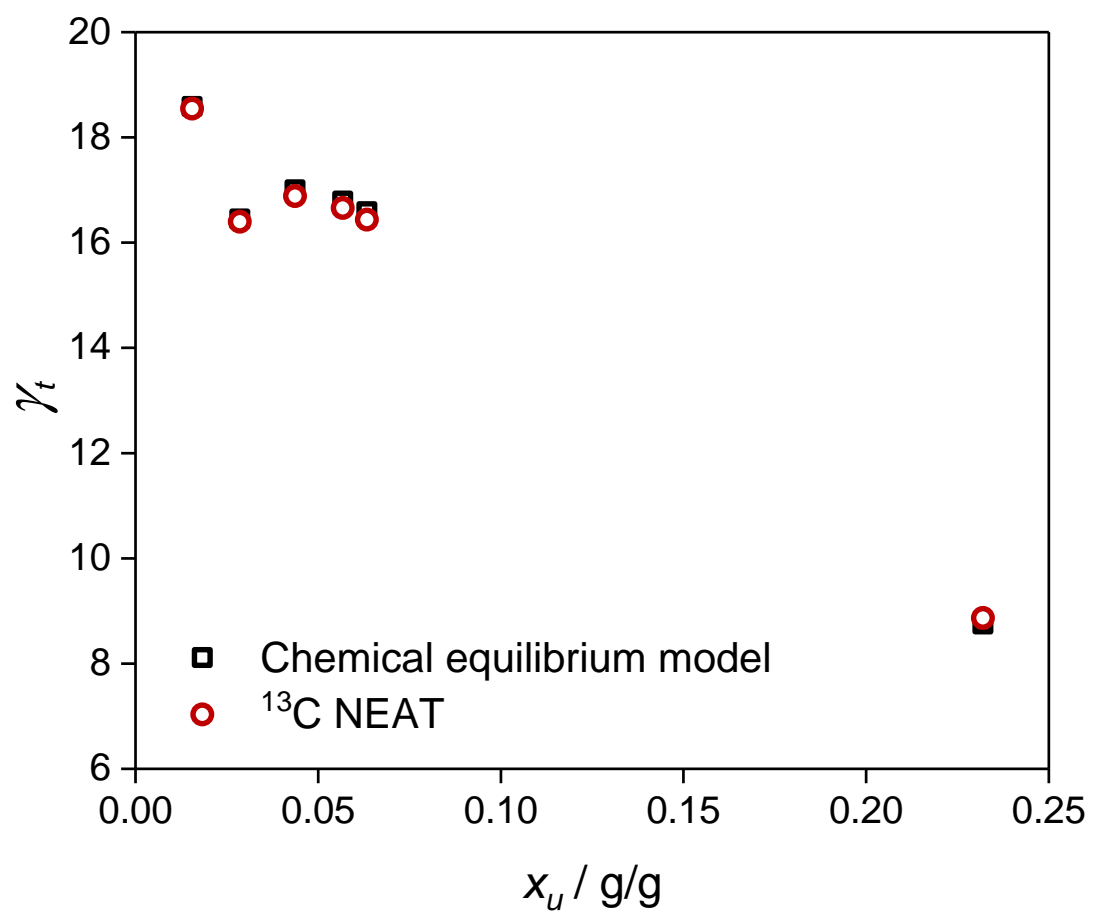

Figure 4: Activity coefficient $\gamma_{t}$ of target component $(t=$ dimethoxymethane) in reactive mixtures of $t$ with water $(\mathrm{w})$ and the unknown component ( $u=$ formaldehyde) as a function of the overall mass fraction of formaldehyde $x_{u}$. Black squares: results from a model based on the true speciation of the mixtures, cf. Kuhnert et al. ${ }^{25}$ Red circles: predictions with NEAT based on ${ }^{13} \mathrm{C}$ NMR and only information on $t$.

mixtures. This model is based on an extended version of original UNIFAC ${ }^{12,13}$, which was adapted to mixtures of this type. In the present work, this model was used to calculate the true compositions and the activity coefficients of the components in the reactive mixtures in chemical equilibrium (for the overall and true compositions of the studied mixtures, see Supporting Information). In Figure 4, the results for the activity coefficient of $t$ calculated with this model including the consideration of all relevant reactions are depicted as black squares. The predictions with NEAT based on a ${ }^{13} \mathrm{C}$ NMR spectrum of each mixture in chemical equilibrium are depicted as red circles. For the predictions, only information on $t$ but no information on the chemical reactions, the reaction products, and the composition of the mixtures in chemical equilibrium was used. In this case, NEAT is based on the extended version of original UNIFAC from Kuhnert et al. ${ }^{25}$ The agreement of the predictions with NEAT with the results for the fully specified mixtures is excellent, even at high overall mass 
fractions of formaldehyde.

Another example for the application of NEAT to reactive aqueous mixtures is shown in Figure 5 . The studied mixtures contain the target component ( $t=$ dimethoxymethane) as well

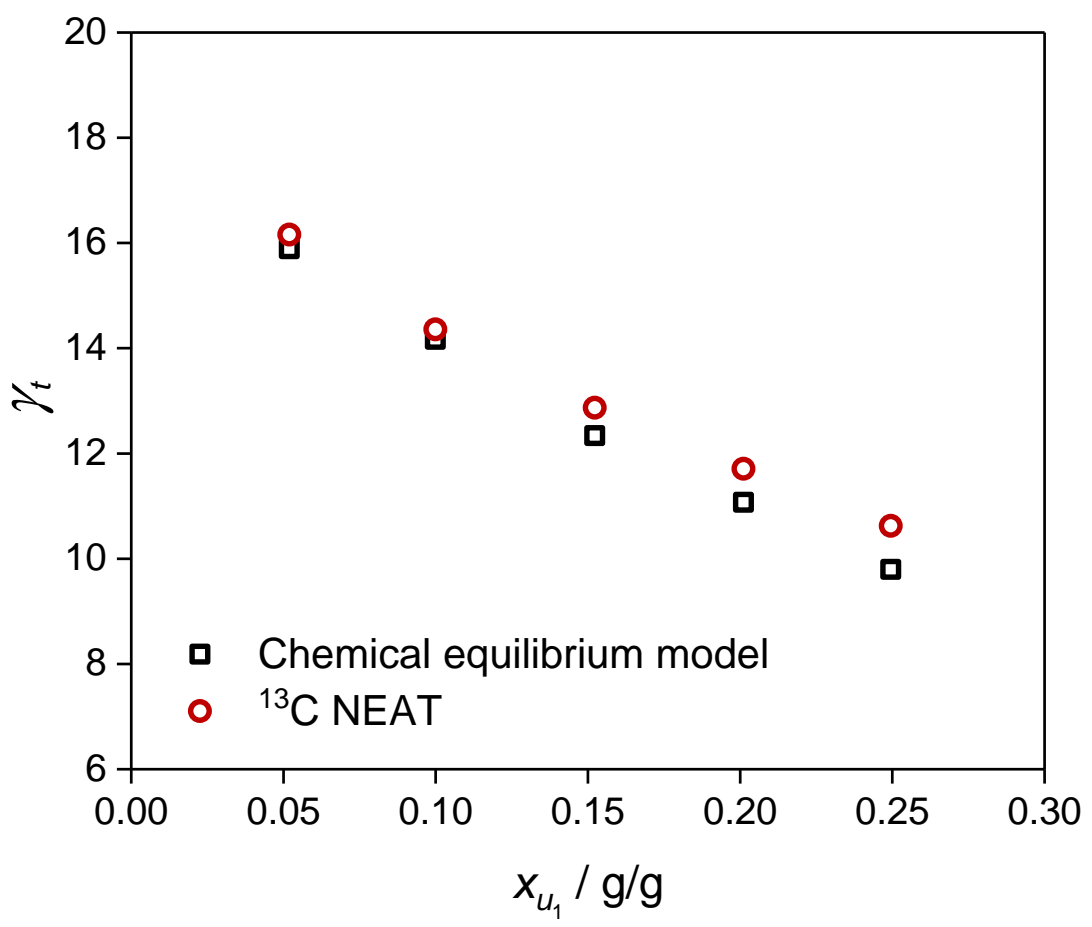

Figure 5: Activity coefficient $\gamma_{t}$ of target component ( $t=$ dimethoxymethane) in reactive mixtures of $t$ with water (w), and two unknown components $\left(u_{1}=\right.$ formaldehyde, $u_{2}=$ methanol) as a function of the overall mass fraction of formaldehyde $x_{u_{1}}$. Black squares: results from a model based on the true speciation of the mixtures, cf. Kuhnert et al. ${ }^{25}$ Red circles: predictions with NEAT based on ${ }^{13} \mathrm{C}$ NMR and only information on $t$.

as water $(\mathrm{w})$ and the unknown components $(u=$ formaldehyde, methanol) in different overall mass fractions. In mixtures of this type, formaldehyde forms polyoxymethylene glycols by reacting with water and polyoxymethylene hemiformals by reacting with methanol. ${ }^{25-29}$ Hence, to calculate the true compositions of these mixtures, again a chemical reaction scheme has to be considered. Again, the model of Kuhnert et al. ${ }^{25}$ was used to calculate the true compositions as well as the activity coefficients of the target component in the studied mixtures (for the overall and true compositions of the mixtures, see Supporting Information). The results based on the full speciation of the mixtures are depicted as black squares in Figure 5. The predictions with NEAT based on a ${ }^{13} \mathrm{C}$ NMR spectrum of each mixture in 
chemical equilibrium are depicted as red circles. Again, for the predictions with NEAT, only information on $t$ but no information on the chemical reactions, the reaction products, and the composition of the mixtures in chemical equilibrium was used. As in the previous example, NEAT is thereby based on the extended version of original UNIFAC from Kuhnert et al. ${ }^{25}$ Also for these complex reactive mixtures, the predictions with NEAT are in very good agreement with the results for the fully specified mixtures.

In a previous work of our group, several examples for the application of NEAT to aqueous poorly specified mixtures were presented. ${ }^{18}$ These predictions were obtained using information on the water mass fraction in the mixtures besides information on the target component. In the Supporting Information, it is shown that NEAT also gives excellent predictions for the activity coefficient of the target component in these mixtures without using information on the water mass fraction, i.e. based only on information on the target component.

\section{Non-aqueous mixtures}

For applying NEAT to non-aqueous organic mixtures, both ${ }^{13} \mathrm{C}$ NMR spectroscopy and ${ }^{1} \mathrm{H}$ NMR spectroscopy were used. The assignment of chemical groups to chemical shift regions used in the present work for both NMR methods are summarized in Tables 3 and 4, respectively. For each NMR method, two variants were studied in this work: predictions using only information on the target component $t$ and predictions using information on $t$ as well as on the nature of a single additional component, referred to as solvent $s$ in the following. However, no information on the concentration of $s$ was used.

In Figure 6, results for the activity coefficient of the target component $(t=$ ethanol) in five-component mixtures containing different concentrations of the unknown components ( $u=$ oleic acid, tert-butylhydroquinone, acetone, 1-propanol) are shown.

The black squares show the predictions with NEAT based on ${ }^{13} \mathrm{C}$ NMR if only information on $t$ is used. The trend of the results calculated with UNIFAC for the fully specified mixtures, which are shown as line in Figure 6, is well represented by these predictions. How- 


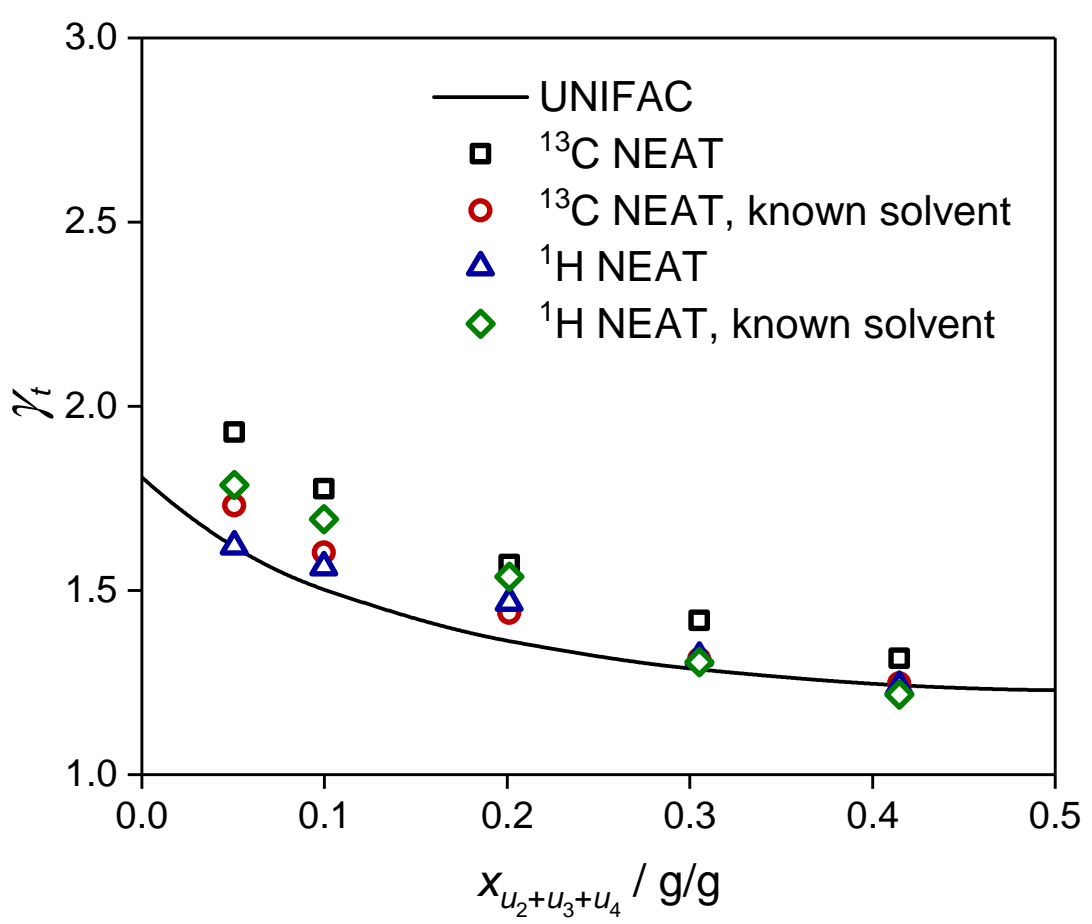

Figure 6: Activity coefficient $\gamma_{t}$ of target component ( $t=$ ethanol) in five-component mixtures with four unknown components $\left(u_{1}=\right.$ oleic acid, $u_{2}=$ tert-butylhydroquinone (TBHQ), $u_{3}=$ acetone $u_{4}=1$-propanol) as a function of the total mass fraction of $u_{2}, u_{3}$, and $u_{4}$ at $298 \mathrm{~K}$ and 1 bar. The mass ratio of $\left(t: u_{1}\right)$ is $(0.051: 0.949)$, the mass ratio of $\left(u_{2}: u_{3}: u_{4}\right)$ is $(0.340: 0.331: 0.329)$ in all mixtures. Line: results from modified UNIFAC (Dortmund) for the fully specified mixtures. Black squares: predictions with NEAT based on ${ }^{13} \mathrm{C}$ NMR and only information on $t$. Red circles: predictions with NEAT based on ${ }^{13} \mathrm{C}$ NMR and information on $t$ and the nature of $u_{1}$, i.e. $s=u_{1}$. Blue triangles: predictions with NEAT based on ${ }^{1} \mathrm{H}$ NMR and only information on $t$. Green diamonds: predictions with NEAT based on ${ }^{1} \mathrm{H}$ NMR and information on $t$ and the nature of $u_{1}$, i.e. $s=u_{1}$.

ever, the activity coefficient of $t$ is overestimated. The red circles show the predictions with NEAT that are also based on ${ }^{13} \mathrm{C}$ NMR but use information on $t$ and, additionally, on oleic acid, which is considered as the known solvent $s$. Using additional information on $s$ leads to a better accuracy of the predictions with ${ }^{13} \mathrm{C}$ NEAT and a very good agreement with the results for the fully specified mixtures. The predictions with NEAT based on ${ }^{1} \mathrm{H}$ NMR and only information on $t$, which are denoted as blue triangles in Figure 6, show a very good agreement with the results for the fully specified mixtures. Using additional information on the known solvent $s$ does not lead to a significant improvement of the predictions with ${ }^{1} \mathrm{H}$ NEAT, as can be seen by the green diamonds in Figure 6 . 
In Figure 7, results for the influence of the value of the molar mass $M_{\tilde{u}}$ of the mean unknown component $\tilde{u}$ on the predictions with NEAT are shown. As an example, one mixture with the target component $(t=$ ethanol) from Figure 6 was selected for this purpose. Similar to

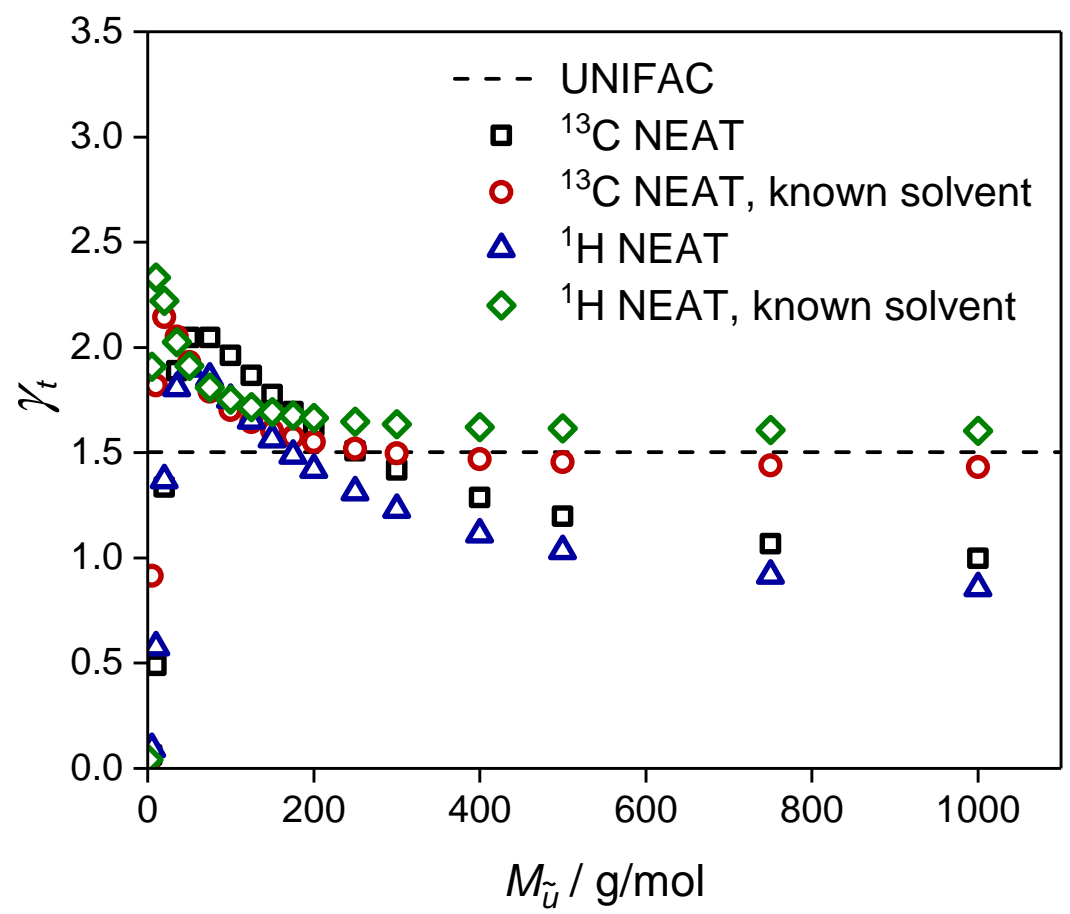

Figure 7: Activity coefficient $\gamma_{t}$ of target component $(t=$ ethanol) in a five-component mixture with four unknown components $\left(u_{1}=\right.$ oleic acid, $u_{2}=$ tert-butylhydroquinone, $u_{3}$ = acetone, $u_{4}=1$-propanol) as a function of the molar mass $M_{\tilde{u}}$ of the mean unknown component $\tilde{u}$ at $298 \mathrm{~K}$ and 1 bar. The composition of the mixture is $x_{t}=0.046 \mathrm{~g} / \mathrm{g}$, $x_{u_{1}}=0.855 \mathrm{~g} / \mathrm{g}, x_{u_{2}}=0.034 \mathrm{~g} / \mathrm{g}, x_{u_{3}}=0.033 \mathrm{~g} / \mathrm{g}$. Dashed line: result from modified UNIFAC (Dortmund) for the fully specified mixture. Black squares: predictions with NEAT based on ${ }^{13} \mathrm{C}$ NMR and only information on $t$. Red circles: predictions with NEAT based on ${ }^{13} \mathrm{C}$ NMR and information on $t$ and the nature of $u_{1}$, i.e. $s=u_{1}$. Blue triangles: predictions with NEAT based on ${ }^{1} \mathrm{H}$ NMR and only information on $t$. Green diamonds: predictions with NEAT based on ${ }^{1} \mathrm{H}$ NMR and information on $t$ and the nature of $u_{1}$, i.e. $s=u_{1}$.

the results for aqueous mixtures, cf. Figure 2, a strong influence can be observed for values of $M_{\tilde{u}}<50 \mathrm{~g} / \mathrm{mol}$. However, in contrast to the results for aqueous mixtures, a significant influence can be observed also for larger values. For the predictions for which only information on the target component $t$ was used, similar results were found independent on whether ${ }^{13} \mathrm{C}$ or ${ }^{1} \mathrm{H}$ NMR spectroscopy was used, cf. black squares and blue triangles in Figure 7, respectively. Using additional information on the nature of oleic acid, i.e. considering oleic acid as 
the known solvent $s$, leads to a smaller influence of $M_{\tilde{u}}$ on the predictions with NEAT for both NMR methods, especially at values $M_{\tilde{u}}>100 \mathrm{~g} / \mathrm{mol}$. This is in analogy to the results found for the aqueous mixtures, in which water was considered as known solvent. Similar results were found for all studied non-aqueous mixtures. Hence, for considering non-aqueous mixtures, a reasonable estimate for $M_{\tilde{u}}$ is more important than for aqueous mixtures, especially if only information on the target component $t$ but no other component, e.g. the solvent $s$, is available.

Figure 8 shows results for the activity coefficient of the target component $(t=$ ethanol) in five-component mixtures with four unknown components $(u=$ methyl oleate, methyl stearate, methyl palmitate, tert-butylhydroquinone) as a function of the mass fraction of $t$. All predictions with NEAT represent the trend of the results for the fully specified mixtures well. However, using only information on the target component $t$ leads to an overestimation of the activity coefficient with NEAT based on ${ }^{1} \mathrm{H}$ NMR, cf. blue triangles, and an underestimation with NEAT based on ${ }^{13} \mathrm{C}$ NMR, cf. black squares. The predictions obtained with ${ }^{13} \mathrm{C}$ NEAT and using additional information on the nature of the solvent $(s=$ methyl oleate) show an excellent agreement with the results for the fully specified mixtures, cf. red circles. Regarding ${ }^{1} \mathrm{H}$ NEAT, using additional information on $s$ does only lead to a slight improvement of the predictions, cf. green diamonds. As expected, the agreement generally gets better the higher the mass fraction of target component becomes and, thus, the more information on the mixture is available as input for NEAT.

In Figure 9, results for the activity coefficient of the target component ( $t=$ dimethoxymethane) in five-component mixtures with four unknown components $(u=$ methyl oleate, tert-butylhydroquinone, methanol, acetone) are shown.

The trend of the results for the fully specified mixtures is poorly described by the predictions with NEAT based on ${ }^{13} \mathrm{C}$ NMR and only information on $t$, cf. black squares. By considering additional information on the nature of methyl oleate, i.e. by considering methyl oleate as the known solvent $s$, the predictions show a very good agreement with the results 


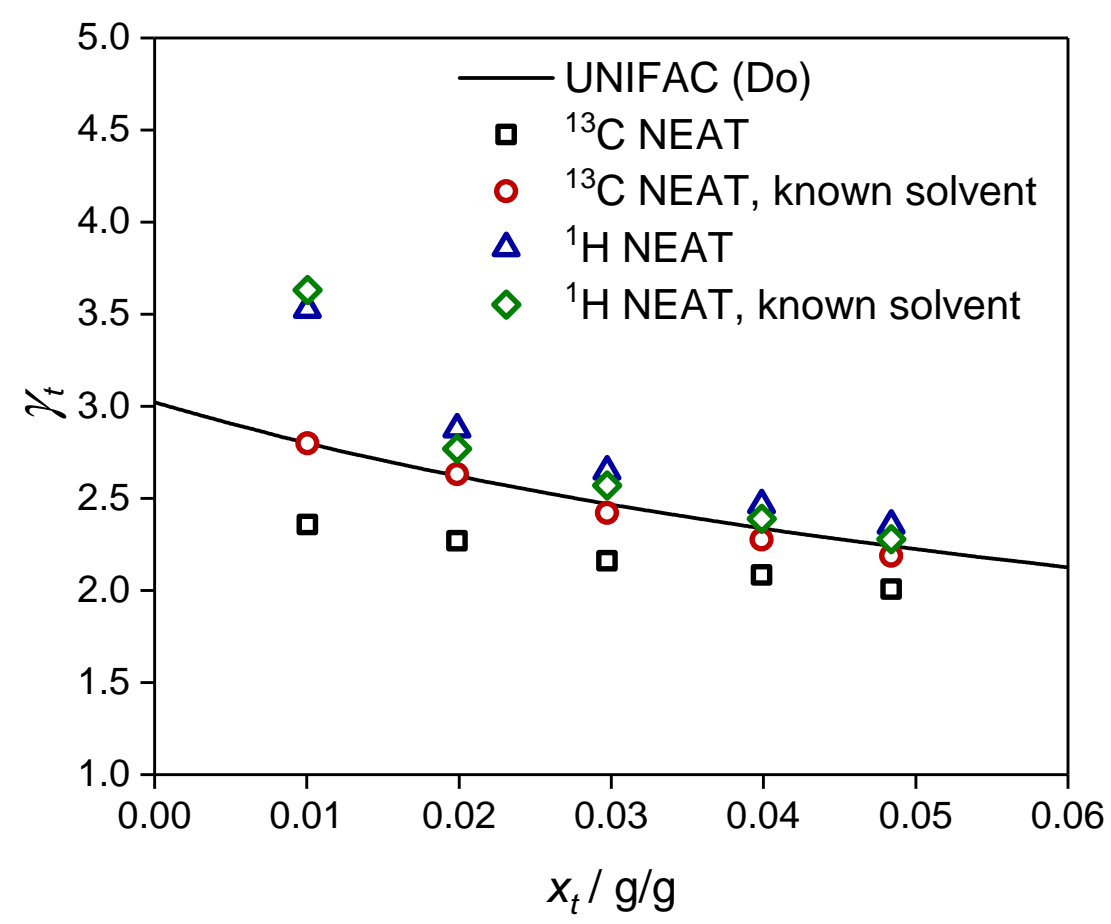

Figure 8: Activity coefficient $\gamma_{t}$ of target component ( $t=$ ethanol) in five-component mixtures with four unknown components $\left(u_{1}=\right.$ methyl oleate, $u_{2}=$ methyl stearate, $u_{3}=$ methyl palmitate, $u_{4}=$ tert-butylhydroquinone) as a function of the mass fraction of $t$ at $298 \mathrm{~K}$ and 1 bar. The mass ratio of $\left(u_{1}: u_{2}: u_{3}: u_{4}\right)$ is $(0.698: 0.145: 0.122: 0.035)$ in all mixtures. Line: results from modified UNIFAC (Dortmund) for the fully specified mixtures. Black squares: predictions with NEAT based on ${ }^{13} \mathrm{C}$ NMR and only information on $t$. Red circles: predictions with NEAT based on ${ }^{13} \mathrm{C}$ NMR and information on $t$ and the nature of $u_{1}$, i.e. $s=u_{1}$. Blue triangles: predictions with NEAT based on ${ }^{1} \mathrm{H}$ NMR and only information on $t$. Green diamonds: predictions with NEAT based on ${ }^{1} \mathrm{H}$ NMR and information on $t$ and the nature of $u_{1}$, i.e. $s=u_{1}$.

for the fully specified mixtures, cf. red circles. A similar good agreement was found for the predictions based on ${ }^{1} \mathrm{H}$ NMR, independent on whether only information on $t$ or additional information on $s$ were used, cf. blue triangles and green diamonds, respectively. 


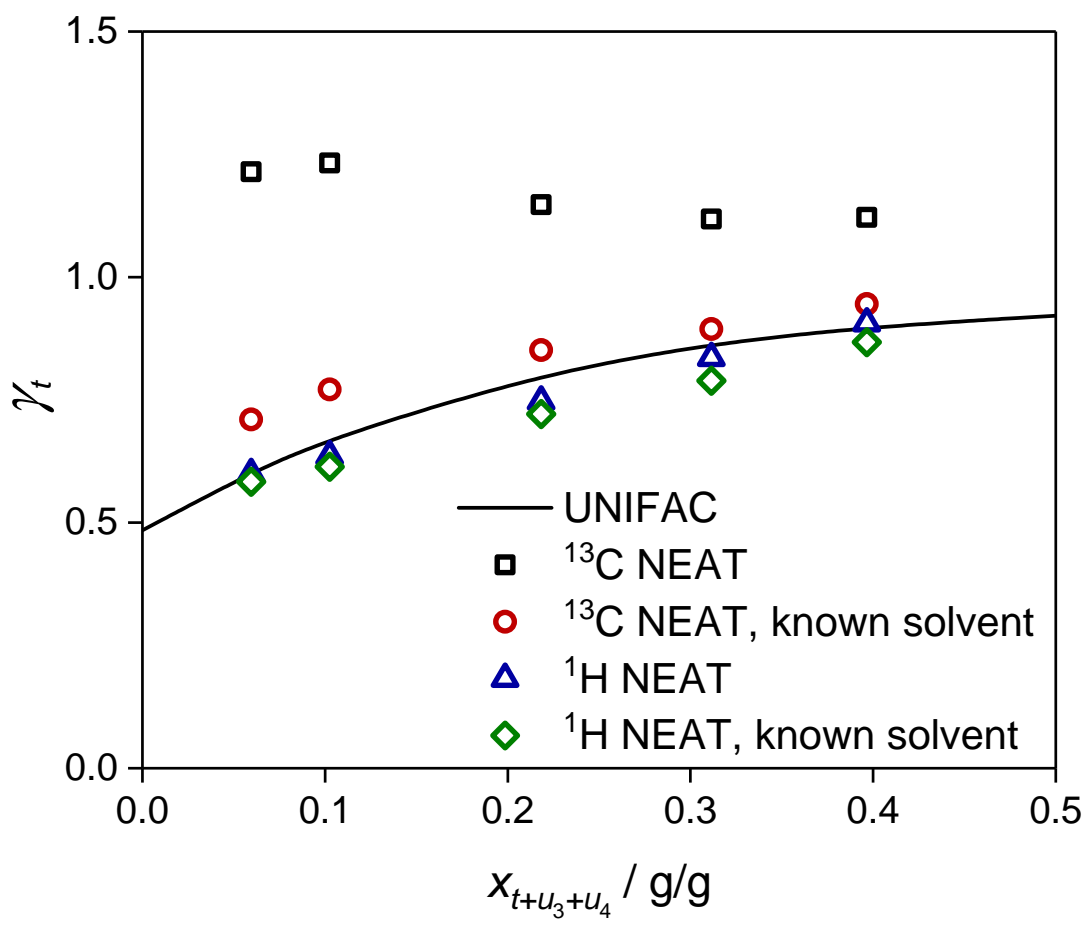

Figure 9: Activity coefficient $\gamma_{t}$ of target component ( $t=$ dimethoxymethane) in fivecomponent mixtures with four unknown components $\left(u_{1}=\right.$ methyl oleate, $u_{2}=$ tertbutylhydroquinone, $u_{3}=$ methanol, $u_{4}=$ acetone) as a function of the total mass fraction of $t, u_{1}$, and $u_{2}$ at $298 \mathrm{~K}$ and 1 bar. The mass ratio of $\left(t: u_{3}: u_{4}\right)$ is $(0.341: 0.337: 0.322)$, the mass ratio of $\left(u_{1}: u_{2}\right)$ is $(0.949: 0.051)$ in all mixtures. Line: results from modified UNIFAC (Dortmund) for the fully specified mixtures. Black squares: predictions with NEAT based on ${ }^{13} \mathrm{C}$ NMR and only information on $t$. Red circles: predictions with NEAT based on ${ }^{13} \mathrm{C}$ NMR and information on $t$ and the nature of $u_{1}$, i.e. $s=u_{1}$. Blue triangles: predictions with NEAT based on ${ }^{1} \mathrm{H}$ NMR and only information on $t$. Green diamonds: predictions with NEAT based on ${ }^{1} \mathrm{H}$ NMR and information on $t$ and the nature of $u_{1}$, i.e. $s=u_{1}$ 


\section{Conclusions}

Liquid mixtures that contain a known target component but are otherwise poorly specified are common in process engineering. The conceptual process design in this situation is challenging since the existing thermodynamic models cannot be applied to such mixtures. In a previous work of our group, a method to estimate the activity coefficients of target components in such poorly specified mixtures was presented. The method is called NEAT and is based on a combination of NMR spectroscopy, which yields information on chemical groups in the mixture, and a thermodynamic group contribution method to calculate activity coefficients. Although the approach of NEAT is based on groups rather than on components, it can be interpreted as a physically motivated way of specifying such pseudo components. In the present work, NEAT is described in more detail than in the previous short communication. ${ }^{18}$ In particular, also the mathematical equations are given. In the previous work, NEAT based on results from ${ }^{13} \mathrm{C}$ NMR spectroscopy was applied to some aqueous nonreacting mixtures to demonstrate the applicability of the method. That work was extended here to non-aqueous and reactive mixtures. It is furthermore shown that NEAT also works if only information on the target component is available and no information on the solvent. However, it is shown that the consideration of information on additional components, i.e. the

nature of the solvent, generally improves the predictions with NEAT. Furthermore, ${ }^{1} \mathrm{H}$ NMR spectroscopy was used together with NEAT for the first time. Overall, the results from the present study show that NEAT is a robust method to predict the activity coefficients of target components in poorly specified mixtures with high accuracy and therefore is a valuable tool for the conceptual process design regarding such mixtures. NEAT is highly flexible and can be tailored to specific tasks and situations, e.g. by choosing the most suitable analytical method or thermodynamic model, or by considering additional available information. In future work, NEAT will be extended to other thermodynamic models, such as COSMO-RS, and other NMR-spectroscopic techniques, and to predicting phase equilibria in systems with poorly specified mixtures. 


\section{Acknowledgement}

The authors gratefully acknowledge financial support from the European Unions's Horizon 2020 research and innovation program under grant agreement number 636077.

\section{Supporting Information Available}

The following files are available free of charge:

- Flowchart of NEAT procedure

- Composition of formaldehyde-containing mixtures

- Assignment of groups to components

- Additional results for aqueous poorly specified mixtures

\section{Nomenclature}

\section{Symbols}

$\begin{array}{ll}a & \text { binary group interaction parameter } \\ A_{p} & \text { relative area under signal } p \\ b & \text { molality }[\mathrm{mol} / \mathrm{kg}] \\ c & \text { molarity }[\mathrm{mol} / \mathrm{l}] \\ g & \text { chemical group } \\ G & \text { total number of chemical groups } \\ i & \text { component } \\ k & \text { known component } \\ K & \text { total number of known components } \\ m & \text { mass }[\mathrm{g}]\end{array}$




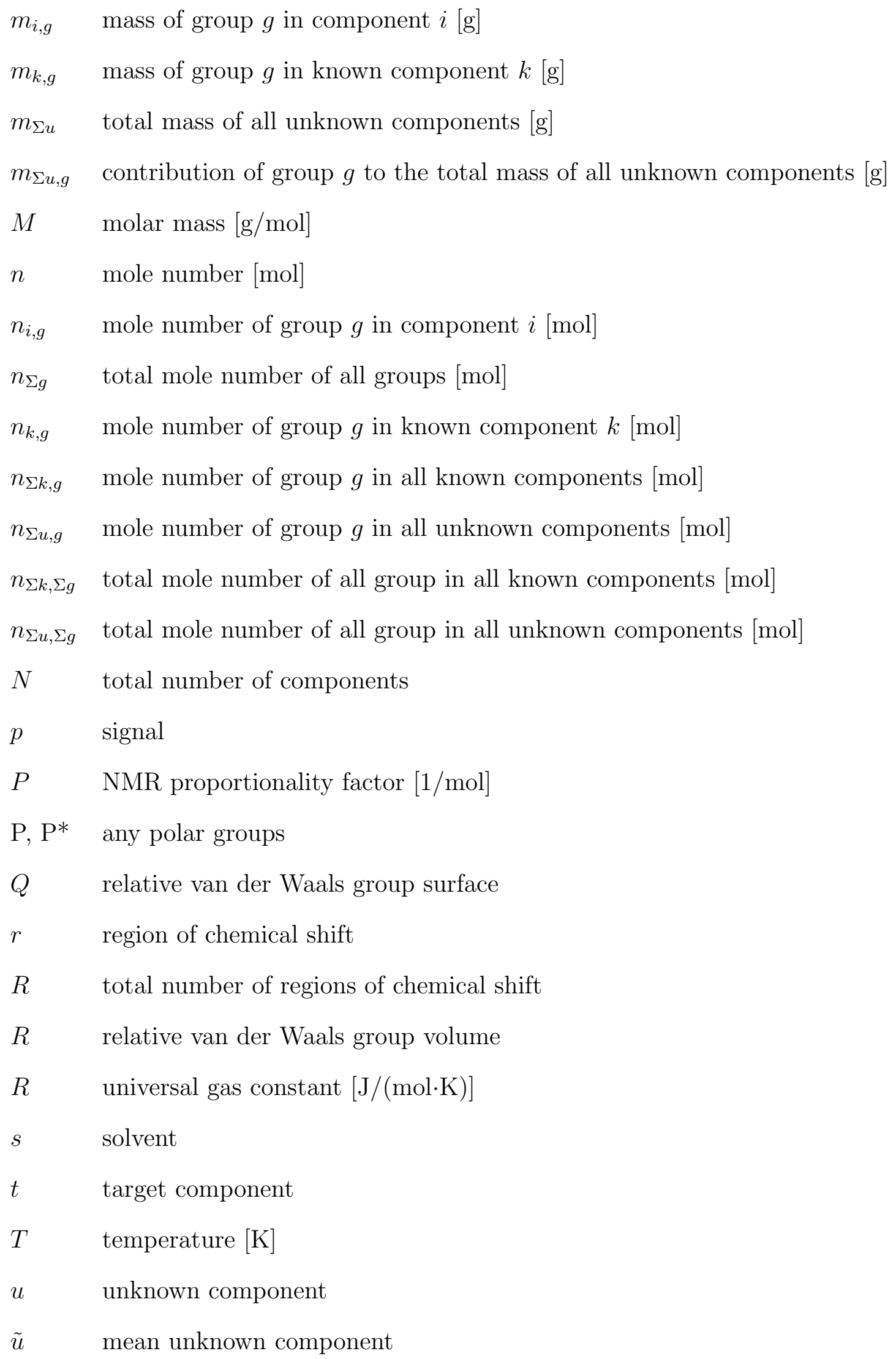




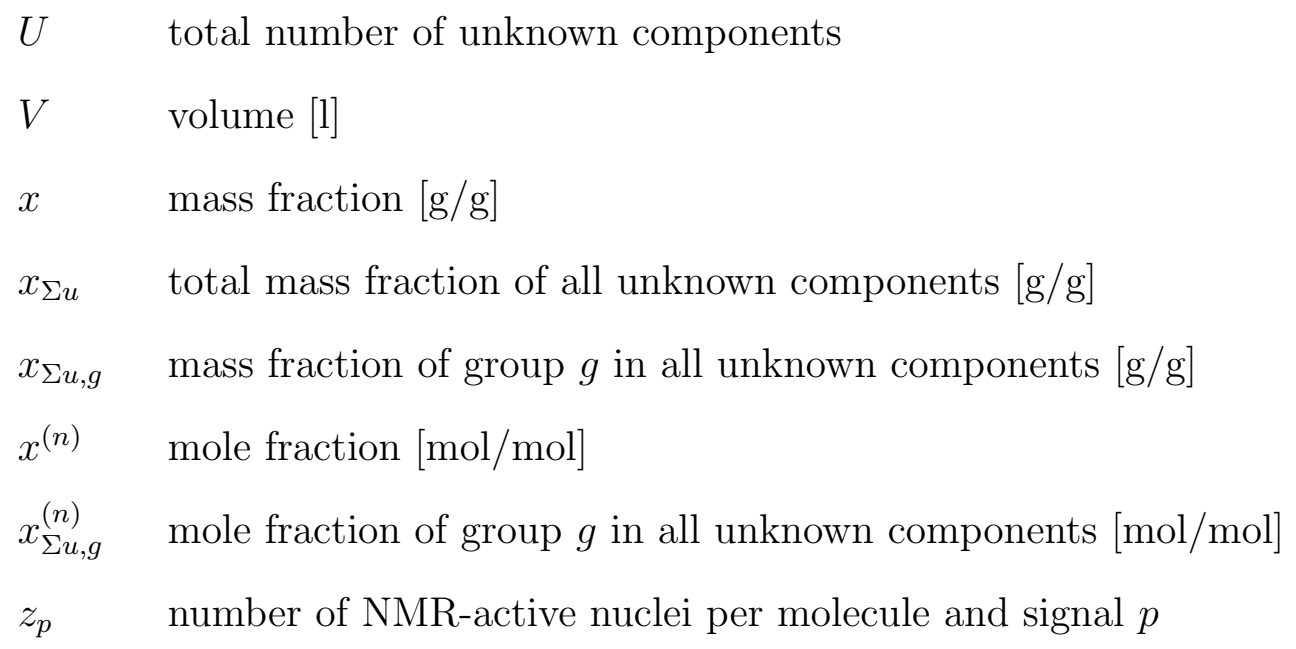

\section{Greek letters}

$\gamma \quad$ activity coefficient

$\Delta \quad$ difference

$\mu \quad$ chemical potential $[\mathrm{J} / \mathrm{mol}]$

$\nu_{i, g} \quad$ stoichiometric coefficient of group $g$ in component $i$

$\nu_{k, g} \quad$ stoichiometric coefficient of group $g$ in known component $k$

$\nu_{t, g} \quad$ stoichiometric coefficient of group $g$ in target component $t$

$\nu_{\tilde{u}, g} \quad$ stoichiometric coefficient of group $g$ in mean unknown component $\tilde{u}$

\section{Sub- and superscripts}


* normalized according to Henry's law on the mole fraction scale

$*, b$ normalized similar to Henry's law on the molality scale

*, c normalized similar to Henry's law on the molarity scale

0 reference state: pure liquid component

$0, \mathrm{H}$ reference state: infinite dilution on the mole fraction scale

$0, b$ reference state: infinite dilution on the molality scale

$0, c$ reference state: infinite dilution on the molarity scale

C combinatorial part

$\mathrm{R}$ residual part

\section{Abbreviations}

COSMO-RS Conductor like Screening Model for Real Solvents IR Infrared

NEAT NMR Spectroscopy for the Estimation of Activity Coefficients of Target Components in Poorly Specified Mixtures

NMR Nuclear Magnetic Resonance

NRTL Non-Random Two-Liquid

TMSP Sodium 3-(trimethylsilyl)tetradeuteriopropionate

UNIFAC Universal Quasichemical Functional Group Activity Coefficients

UNIQUAC Universal Quasichemical

water

\section{References}

(1) Alexander, G. L.; Creagh, A. L.; Prausnitz, J. M. Phase Equilibria for High-Boiling Fossil-Fuel Distillates. 1. Characterization. Industrial \& Engineering Chemistry Fundamentals 1985, 24, 301-310. 
(2) Alexander, G. L.; Schwarz, B. J.; Prausnitz, J. M. Phase Equilibria for High-Boiling Fossil-Fuel Distillates. 2. Correlation of Equation-of-State Constants with Characterization Data for Phase-Equilibrium Calculations. Industrial \&3 Engineering Chemistry Fundamentals 1985, 24, 311-315.

(3) Hartounian, H.; Allen, D. T. Group Contribution Methods for Coal-derived Liquids: Hydrogen Solubilities using a UNIFAC Approach. Fuel 1988, 67, 1609-1614.

(4) Fahim, M. A.; Elkilani, A. S. Prediction of Solubility of Hydrogen in Petroleum Cuts using Modified UNIFAC. The Canadian Journal of Chemical Engineering 1992, 70, $335-340$.

(5) Darwish, E.; Al-Sahhaf, T. A.; Fahim, M. A. Prediction of the Surface Tension of Petroleum Cuts using a Modified UNIFAC Group Contribution Method. Fluid Phase Equilibria 1995, 105, 229-239.

(6) Carreon-Calderon, B.; Uribe-Vargas, V.; Ramirez-Jaramillo, E.; Rairez-de Santiago, M. Thermodynamic Characterization of Undefined Petroleum Fractions using Group Contribution Methods. Industrial \& Engineering Chemistry Research 2012, 51, 1418814198.

(7) Abdul Jameel, A. G.; Elbaz, A. M.; Emwas, A.-H.; Roberts, W. L.; Sarathy, S. M. Calculation of Average Molecular Parameters, Functional Groups, and a Surrogate Molecule for Heavy Fuel Oils Using 1H and 13C Nuclear Magnetic Resonance Spectroscopy. Energy \& Fuels 2016, 30, 3894-3905.

(8) Allen, D. T.; Gray, M. R.; Le, T. T. Structural Characterization and Thermodynamic Property Estimation for Wood Tars: A Functional Group Approach. Liquid Fuels Technology 1984, 2, 327-353.

(9) Renon, H.; Prausnitz, J. M. Local Compositions in Thermodynamic Excess Functions for Liquid Mixtures. AIChE Journal 1968, 14, 135-144. 
(10) Abrams, D. S.; Prausnitz, J. M. Statistical Thermodynamics of Liquid Mixtures: A New Expression for the Excess Gibbs Energy of Partly or Completely Miscible systems. AIChE Journal 1975, 21, 116-128.

(11) Maurer, G.; Prausnitz, J. M. On the Derivation and Extension of the UNIQUAC Equation. Fluid Phase Equilibria 1978, 2, 91-99.

(12) Fredenslund, A.; Jones, R. L.; Prausnitz, J. M. Group-contribution Estimation of Activity Coefficients in Nonideal Liquid Mixtures. AIChE Journal 1975, 21, 1086-1099.

(13) Fredenslund, A.; Gmehling, J.; Rasmussen, P. Vapor-Liquid Equilibria using UNIFAC, a Group-Contribution Method; Elsevier: Amsterdam, The Netherlands, 1977.

(14) Weidlich, U.; Gmehling, J. A Modified UNIFAC Model. 1. Prediction of VLE, $\mathrm{h}^{\mathrm{E}}$, and $\gamma^{\infty}$. Industrial \& Engineering Chemistry Research 1987, 26, 1372-1381.

(15) Constantinescu, D.; Gmehling, J. Further Development of Modified UNIFAC (Dortmund): Revision and Extension 6. Journal of Chemical \&3 Engineering Data 2016, 61, 2738-2748.

(16) Klamt, A. Conductor-Like Screening Model for Real Solvents: A New Approach to the Quantitative Calculation of Solvation Phenomena. The Journal of Physical Chemistry 1995, 99, 2224Ü2235.

(17) Klamt, A.; Jonas, V.; Bürger, T.; Lohrenz, J. C. W. Refinement and Parametrization of COSMO-RS. The Journal of Physical Chemistry A 1998, 102, 5074-5085.

(18) Jirasek, F.; Burger, J.; Hasse, H. Method for Estimating Activity Coefficients of Target Components in Poorly Specified Mixtures. Industrial \& Engineering Chemistry Research 2018, 57, 7310-7313.

(19) Mu, T.; Rarey, J.; Gmehling, J. Group Contribution Prediction of Surface Charge Density Profiles for COSMO-RS(Ol). AIChE Journal 2007, 53, 3231-3240. 
(20) Scheithauer, A.; Grützner, T.; Rijksen, C.; Zollinger, D.; Thiel, W.; von Harbou, E.; Hasse, H. 1H- and 13C-NMR-Spectroscopic Study of Chemical Equilibria in the System Acetaldehyde + Water. AIChE Journal 2015, 61, 177-187.

(21) Steinhof, O.; Scherr, G.; Hasse, H. Investigation of the Reaction of 1,3-Dimethylurea with Formaldehyde by Quantitative On-Line NMR Spectroscopy: A Model for the Urea-Formaldehyde System. Magnetic Resonance in Chemistry 2016, 54, 457-476.

(22) Behrens, R.; von Harbou, E.; Thiel, W.; Böttinger, W.; Ingram, T.; Sieder, G.; Hasse, H. Monoalkylcarbonate Formation in Methyldiethanolamine-H2O-CO2. Industrial $\&$ Engineering Chemistry Research 2017, 56, 9006-9015.

(23) Maiwald, M.; Grützner, T.; Ströfer, E.; Hasse, H. Quantitative NMR Spectroscopy of Complex Technical Mixtures using a Virtual Reference: Chemical Equilibria and Reaction Kinetics of Formaldehyde-Water-1,3,5-Trioxane. Analytical and Bioanalytical Chemistry 2006, 385, 910-917.

(24) Galeotti, N.; Burger, J.; Hasse, H. Vapor-liquid Equilibrium in the Ternary Systems Acetic Acid + Water + (Xylose or Glucose). Fluid Phase Equilibria 2018, 473, 323-329.

(25) Kuhnert, C.; Albert, M.; Breyer, S.; Hahnenstein, I.; Hasse, H.; Maurer, G. Phase Equilibrium in Formaldehyde Containing Multicomponent Mixtures: Experimental Results for Fluid Phase Equilibria of (Formaldehyde + (Water or Methanol) + Methylal)) and (Formaldehyde + Water + Methanol + Methylal) and Comparison with Predictions. Industrial \& Engineering Chemistry Research 2006, 45, 5155-5164.

(26) Hasse, H.; Hahnenstein, I.; Maurer, G. Revised Vapor-liquid Equilibrium Model for Multicomponent Formaldehyde Mixtures. AIChE Journal 1990, 36, 1807-1814.

(27) Hahnenstein, I.; Hasse, H.; Kreiter, C. G.; Maurer, G. 1H- and 13C-NMR-Spectroscopic Study of Chemical Equilibria in Solutions of Formaldehyde in Water, Deuterium Oxide, and Methanol. Industrial \& Engineering Chemistry Research 1994, 33, 1022-1029. 
(28) Maiwald, M.; Fischer, H. H.; Ott, M.; Peschla, R.; Kuhnert, C.; Kreiter, C. G.; Maurer, G.; Hasse, H. Quantitative NMR Spectroscopy of Complex Liquid Mixtures: Methods and Results for Chemical Equilibria in Formaldehyde-Water-Methanol at Temperatures up to 383 K. Industrial \& Engineering Chemistry Research 2003, 42, 259-266.

(29) Schmitz, N.; Homberg, F.; Berje, J.; Burger, J.; Hasse, H. Chemical Equilibrium of the Synthesis of Poly(oxymethylene) Dimethyl Ethers from Formaldehyde and Methanol in Aqueous Solutions. Industrial \& Engineering Chemistry Research 2015, 54, 6409-6417. 


\section{Graphical TOC Entry}

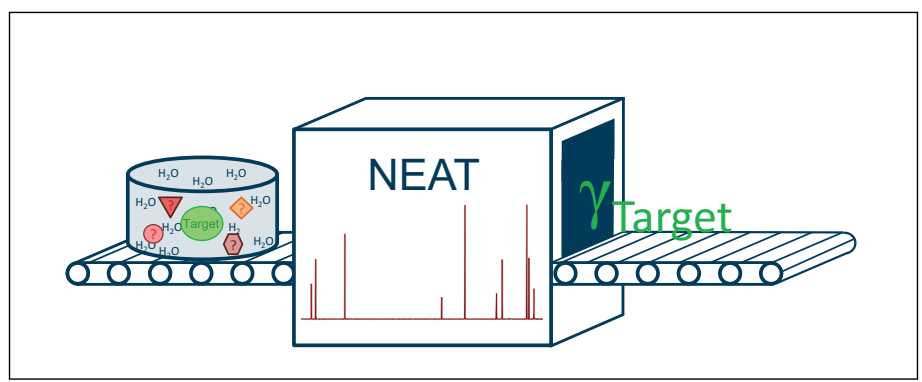

